
\title{
3 Research Square \\ Bioinformatics-based Screening of Key Genes for Saponin Metabolism in Quinoa
}

\section{Chengang Guo}

Dezhou University

\section{Zhimin wei}

Institute of Millet Crops, Hebei Academy of Agriculture and Forestry Sciences, Cereal Crops Research Laboratory of Hebei Province, National Foxtail Millet Improvement Center

Wei Lyu (D Ivwei91@126.com)

National Semi-Arid Agriculture Engineering Technology Research Center

\section{Yanlou Geng}

National Semi-Arid Agriculture Engineering Technology Research Center

\section{Research Article}

Keywords: Quinoa saponins, physiologic , genes, gene set enrichment analysis (GSEA) , principal component analysis (PCA)

Posted Date: January 7th, 2021

DOl: https://doi.org/10.21203/rs.3.rs-139481/v1

License: (c) (1) This work is licensed under a Creative Commons Attribution 4.0 International License. Read Full License 


\section{Abstract}

Quinoa saponins have complex, diverse and evident physiologic activities. However, the key regulatory genes for quinoa saponin metabolism are not yet well studied. The purpose of this study was to explore genes closely related to quinoa saponin metabolism. In this study, the significantly differentially expressed genes in yellow quinoa were firstly screened based on RNA-seq technology. Then, the key genes for saponin metabolism were selected by gene set enrichment analysis (GSEA) and principal component analysis (PCA) statistical methods. Finally, the specificity of the key genes was verified by hierarchical clustering. The results of differential analysis showed that 1654 differentially expressed genes were achieved after pseudogenes deletion. Therein, there were 142 long non-coding genes and 1512 protein-coding genes. Based on GSEA analysis, 116 key candidate genes were found to be significantly correlated with quinoa saponin metabolism. Through PCA dimension reduction analysis, 57 key genes were finally obtained. Hierarchical cluster analysis further demonstrated that these key genes can clearly separate the four groups of samples. The present results could provide references for the breeding of sweet quinoa and would be helpful for the rational utilization of quinoa saponins.

\section{Introduction}

Quinoa (Chenopodium quinoa) has been widely cultivated in South America for 5,000 to 7,000 years ${ }^{1}$. Quinoa's nutritional features have attracted the attention of many scientific researchers. Meanwhile, quinoa's medicinal value has been paid increasing attention and developed ${ }^{2-4}$. Quinoa saponins (QSs) is a bioactive molecule with anti-obesity ${ }^{5}$, anti-cancer ${ }^{6}$, immune response ${ }^{7}$, anti-diabetes ${ }^{8}$, cholesterol- $^{2}$ lowering ${ }^{9}$, anti-inflammation ${ }^{10}$, anti-oxidation ${ }^{11}$ and other medical effects. At the same time, it is a kind of anti-nutrition factor with a slightly bitter and astringent taste ${ }^{12}$, and potentially toxic ${ }^{13}$, which hinders the application of quinoa in food, processing and promotion. The removal, extraction and maximum utilization of quinoa saponins have been a difficult problem for researchers. Therefore, reasonable regulation of saponin content in quinoa products can greatly improve the quality of quinoa food and the efficacy of quinoa drugs, which are of great significance for development of quinoa resources. It is an important goal of quinoa breeding to develop the saponin-free quinoa with good taste and improve the bioactivity utilization rate of QSs.

In the past 30 years, the research on quinoa saponins mainly focuses on the separation, extraction and detection of saponins, structure elucidation and biological activity identification. For example, Verza et al. ${ }^{7}$ extracted and purified quinoa saponins using hydrochloric acid ethanol solution, and used ultra-high performance liquidity-Quadrupole time-of-flight mass spectrometry (UPLC/Q-TOF-MS) qualitatively identified 10 saponins. And their humoral and cellular immune reactivity was confirmed in mice. Gee et al. ${ }^{14}$ assessed the effect of processing technology on saponins content and composition in quinoa products, and studied the biological effects of quinoa grains and cereal products containing high saponins and low levo-cholesterol in vitro in rats. It was found that processed quinoa reduced saponin concentration and membrane solubility activity, and improved palatability and nutritional quality of 
cereals to levels similar to those of wheat-based cereals. León-Roque et al. ${ }^{15}$ developed and verified a microtiter macrolens-coupled smart phone (MCS) assay for quantifying total saponins in quinoa, which is a green and fast analytical method. More and more saponins are extracted and their molecular structure is elucidated, which lays a foundation for the study of their metabolic mechanism. The discovery and elucidating structure of quinoa saponins has laid a foundation for the further study of the metabolic mechanism of quinoa saponins.

Previous studies have shown that the main pathway of mevalonic acid (MVA) in plants produces biosynthetic precursor for the triterpene saponins ${ }^{16,17}$. Squalene synthase, Squalene epoxide cyclase, Cytochrome P450 Monooxygenase (CYP450) were the key enzymes in the biosynthesis of QSs ${ }^{18-20}$. Although the saponin metabolic pathways are relatively clear in the other plants, the study of the key genes involved in the biosynthesis of saponins in quinoa is still in its infancy. The biosynthetic pathway of QSs and its related enzymes have not been fully elucidated ${ }^{21}$. In recent three years, with the development of high-throughput sequencing technology, the metabolic mechanism of quinoa saponins has been intensively studying at the molecular level. The research on key genes of QSs has a certain breakthrough. In 2016, Fiallos-Jurado et al. ${ }^{21}$ screened the genes related to the metabolism of QSs through RNA-seq technology, and identified three key genes (CqbAS1, CqCYP716A78 and CqCYP716A79) through expression in yeast. In 2017, a large international led by Tester ${ }^{22}$ mapped quinoa's complex $1.5 \mathrm{Mb}$ genome using a series of sequencing strategies, identifying and annotating 43 different saponins in the seeds. These important results have been encouraging scientists to design strains with low saponins. In the same year, Zhu et al. generated a high-quality genome draft using an inbred line of the quinoa cultivar Real ${ }^{23}$. The completion of the quinoa genome profiling has marked the introduction of sequencing technology into quinoa research, opening the prelude to the era of quinoa research on big data, which will greatly promote the research on the genetic improvement of quinoa.

The aim of this investigation has been to mine the key genes influencing metabolism of QSs based on RNA-seq technology and statistical methods. This study laid the foundation for the genetic improvement of quinoa, and then should shed new light on the future acquisition of low saponin quinoa varieties through quinoa breeding and genetic engineering.

\section{Materials And Methods}

\section{Acquisition and collation of sequencing data.}

The RNA-seq raw data for four groups of quinoa samples (yellow bitter quinoa seeds ( $\mathrm{Yr}$ ), yellow bitter quinoa flower $(\mathrm{YI})$, white sweet quinoa seeds $(\mathrm{Wr})$, white sweet quinoa flower $(\mathrm{Wl})$, respectively; three biological replicates for each group) are available in the NCBI Sequence Read Archive (SRA), with accession number: SRP226463. The quality control (QC) of the sequencing data has been performed using Fastp ( $v$ 0.19.1) to obtain clean data. Clean data were used for subsequent analysis. Quinoa 


\section{Differential expression analysis.}

Prior to the analysis for finding differentially expressed genes (DEGs), clean reads were aligned to a quinoa reference genome by Hisat2 (v 2.1.0). Gene counts in quantification analysis were computed by featureCounts from Subread ( $v$ 2.0.1). Then differential gene expression analyses were conducted using DESeq (v 1.28.0) package (http://www-huber.embl.de/users/anders/ DESeq/). DEGs were identified based on a corrected $p$ values $($ pad $j)<0.05$ and $\mid \log 2$ foldchange| $>1$

\section{Gene set enrichment analysis.}

Gene set enrichment analysis (GSEA) was carried out with GSEA software (version 1.2), which was obtained from GitHub (https://github.com/GSEA-MSigDB/GSEA_R). The outcome of the differential expression analysis was taken to perform gene set enrichment analysis using the 'preranked' running mode. All the genes in the genome were sorted according to the expression correlation of gene of interest and included in the gene set enrichment analysis. The pre-defined gene set of saponin metabolism pathway is composed of four kind of key genes during saponin metabolism. Enrichment map was used for visualization of the GSEA results. We selected as significant only those enrichments (Normalized Enrichment Score, $|\mathrm{NES}|>1$ ) with a normalized $P$ value $<0.05$, false discovery rate (FDR) $<0.25$ after gene set permutations were performed 1000 times for each analysis.

\section{Principal component analysis (PCA).}

The prcomp function of stats ( $R$ package, version 3.6.1) were used for PCA analyses. PCA analysis was performed using differentially expressed genes for dimensionality reduction and to determine the contribution of genes to each principal component. The genes with |loading valuel $>0.1$ were selected as the final key genes. Based on key genes, R software package pheatmap (https://CRAN.Rproject.org/package=pheatmap) was used to generate a hierarchical cluster heat map and cluster tree.

\section{Gene enrichment analysis.}

The Kyoto Encyclopedia of Genes and Genomes (KEGG) pathway enrichment analysis revealed the significantly enriched pathways of differentially expressed genes analyzed using the enricher function in R software package clusterProfiler (version 3.14.3) ${ }^{24}$. Gene Ontology (GO) term enrichment analysis was performed to provide all the $\mathrm{GO}$ terms that were significantly enriched in the differentially expressed genes using the enrichGO function in clusterProfiler package. 


\section{Results}

\section{Study selection.}

Briefly, six major steps were employed including data collection, data quality control, quantification, differential expression analysis, gene set enrichment analysis, principal component analysis. In the first four steps, we selected the standard transcriptome sequencing analysis to obtain the DEGs. In the fifth step, GSEA was selected to screen genes closely related to saponin metabolism, and the candidate key genes were obtained by taking the intersection with the DEGs. Finally, the key genes were obtained by PCA. The whole study process selection is described in the flowchart (Fig. 1).

\section{Statistics for the differentially expressed genes on the four groups.}

We detected 4013, 19181, 20265 DEGs when comparing the gene expression levels between $\mathrm{Yr}$ and $\mathrm{Wr}$ groups, Yr and YI, Yr and WI respectively. Taking the intersection of three DEGs sets, a total of 1734 significant differentially expressed genes were obtained. The results of differential gene expression analysis were shown in Fig. 2. After further filtering out the pseudogenes, one resulted in a total of 1,654 DEGs.

\section{Screening key candidate genes.}

After differentially analyzing, we obtained DEGs in the seeds of yellow bitter quinoa. To further screen genes closely related to saponin metabolism, we carried out GSEA on all genes in the genome, aiming to screen the genes regulating saponin metabolism pathways. We selected four kind of saponin synthesis key genes as the pre-defined gene set of saponin metabolism pathway. The detailed information of these genes was shown in Table S1. The correlation between gene of interest and other genes was used as the phenotype label. GSEA results showed that 5052 genes were significantly correlated with saponin metabolism. By taking the intersection of the gene sets and that from differential expression results, we obtained 116 key candidate genes (showed in Table S2). Among these, there were 11 long non-coding RNA and 105 protein-encoding genes. Therein, 18 genes are encoding two or more proteins. Fig. 3 lists the top six genes that are closely related to the saponin metabolism pathway based on Normalized Enrichment Score (NES). It can be seen that the genes in the saponin metabolism pathway are significantly enriched to the positive region of the first five genes (Fig. 3 a-e), while they are enriched to the negative region of the sixth gene (Fig. 3f).

\section{Identification of key genes for saponin metabolism.}


To select the key genes from key candidate genes, we performed principal component analysis on 116 key candidate genes (Fig. 4a). As can be seen from Fig. 4a that the first component of the PCA explained $51.92 \%$, while the second component explained $24.63 \%$, of the variance. PC1 significantly distinguished the yellow quinoa seeds from the other three samples. PC2 can distinguished white sweet quinoa seeds from the other two samples (group $\mathrm{Yl}$ and WI). Ultimately, 57 genes were selected as the final key genes. The details of component loadings for each gene are described in Table S3, while the scree plot of PCA is represented in Fig. S1. Details on the expression of 57 genes are presented in Fig. 5.

Hierarchical clustering of 12 samples according to the expression patterns of these 57 key genes suggested that these up-regulated and down-regulated genes could effectively discriminate $\mathrm{Yr}$ and the other three groups. The heatmap and hierarchical clustering were showed in Fig. 4b.

\section{Discussion}

As mentioned in the literature review ${ }^{25,26}$, saponins have high medicinal value. However, QSs have two sides. Despite medicinal value, on the other hand, its bitterness reduces the taste of food, which brings a lot of trouble to the production of quinoa food. To solve this dilemma, many studies have focused on the desaponification treatment of quinoa seeds in food production. With the development of high-throughput sequencing technology, particularly completion of quinoa genome annotation ${ }^{23}$, genomics-assisted breeding has gradually been put on the agenda for the improvement of quinoa varieties. This study was designed to mine the key genes related to QSs metabolism by RNA-seq technologies and several statistical methods. These findings might help others to better understand the metabolic process and regulatory mechanism of QSs. The screened key genes can be used as promising targets for the molecular breeding of improved quinoa, which, in turn, would lay the foundation for the rational utilization of QSs.

The results of differential analysis revealed that there were more DEGs between developmental stages than between breeds. There were 19181 differential genes between the Yr group and YI group (Fig. 2a); 20265 genes between the Yr group and Wl group (Fig. 2c); 4013 genes between the Yr group and Wr group (Fig. 2b). This may be because in addition to the differential expression of metabolic genes, the differences in the expression of structural genes were also highly significant than that between breeds. Through the intersection of three differentially expressed gene sets, most of the differentially expressed structural genes were eliminated, while the differentially expressed metabolic genes were maximally retained. We also carried out KEGG pathway analysis of the screened DEGs and found these genes were significantly enriched in metabolism-related pathways (Fig. S2), which confirmed our speculation.

Gene set enrichment analysis is useful for identifying genes closely related to saponin synthesis. We obtained 116 key candidate genes by performing GSEA. Among 116 genes, 56 genes were up-regulated and 60 were down-regulated between Yr group and Wr group (Table S2). Remarkably, we found that the majority of saponin metabolic pathway genes in Yr group compared to Wr group were down-regulated (Table S1), which was contrary to the up-regulation of saponin content. Consequently, we speculate that 
there are new metabolic pathways for saponin in yellow quinoa seeds in addition to the metabolic pathway to our knowledge. In 57 key genes analyzed by PCA, we found 22 genes encoding uncharacterized proteins closely related to the metabolism of quinoa saponin (marked by asterisks in Fig. 5), which partially corroborates our speculation.

In order to select key genes from 116 genes, principal component analysis was carried out (Fig. 4a). We filtered 57 key genes using PCA. Details about the expression of these genes are displayed in Fig. $4 \mathrm{~b}$ and Fig. 5. As can be seen, of the 57 selected genes, 51 genes were up-regulated and 6 genes were downregulated between $\mathrm{Yr}$ and $\mathrm{Wr}$ group. We speculated that these up-regulated genes were positive regulators of saponin biosynthesis, while down-regulated genes may play a role in negative regulators.

To identify key genes, hierarchical cluster analysis and PCA analysis were performed based on 57 genes. Hierarchical clustering of 12 quinoa samples according to the expression patterns of 57 key genes suggested that these genes could effectively discriminate four groups (Fig. 4b). PCA analysis showed that the expression of 57 key genes explained $84.19 \%$ of the variable (Fig. S3), which better explained the differences of the four groups of samples than that based on 116 genes (Fig. 4a).

There are still some weaknesses of this work. Firstly, we have not identified the 57 key genes in an experimental way. Secondly, in this study, one interesting finding was we also identified 10 IncRNAs. Previous studies have been suggested that, in plants, IncRNAs play important roles as regulators in gene silencing ${ }^{27}$, flowering time control ${ }^{28}$, organogenesis in roots ${ }^{29}$, photomorphogenesis in seedlings ${ }^{30}$, abiotic stress responses ${ }^{31,32}$, and reproduction ${ }^{33}$. What role these 10 IncRNAs play in the regulation of saponin metabolism that we need to explore further. Thirdly, in this study, due to incomplete information on genome annotation, we found some uncharacterized genes (Table S1 and Fig. 5), whose specific functions need to be further verified in the saponin biosynthesis process.

\section{Conclusion}

The main goal of the current study was to mine the genes associated closely with quinoa saponin metabolism. Based on differential expression analysis between groups using RNA-seq technology, 1734 differentially expressed genes were obtained. subsequently, 116 genes strongly related to saponin metabolism were screened using GSEA. Finally, 57 key genes were obtained as a result of PCA dimension reduction analysis. Though several limitations need to be acknowledged, this pilot study is the first genome-wide screening of quinoa saponin metabolism key genes. The findings from this study might help others to better understand the metabolic mechanism of saponins in quinoa and provide a reference for the breeding of low saponin quinoa varieties. With regard to the outstanding issues in the study, further research will be undertaken in the following work.

\section{Declarations}

\section{Acknowledgements}


This work was supported by Special projects to tackle key technologies for high-quality agricultural development (No. 192275270)

\section{Author contributions statement}

Y.G. and W.L. conceived the experiments, G.G. and Z.W. wrote and edited this paper and analyzed the results. All authors reviewed and approved the manuscript.

\section{Competing interests}

The authors declare no competing interests.

\section{References}

1. El Hazzam, K. et al. An Insight Into Saponins From Quinoa (Chenopodium Quinoa Willd): A Review. Molecules (Basel, Switzerland). 25, 1059, DOI: https://doi.org/10.3390/molecules2505105 9 (2020).

2. Woldemichael, G. M. \& Wink, M. Identification and Biological Activities of Triterpenoid Saponins From Chenopodium Quinoa. J Agric Food Chem. 49, 2327-2332, DOI: https://doi.org/10.1021/ jf0013499 (2001).

3. Verza, S. G. et al. Immunoadjuvant Activity, Toxicity Assays, and Determination by UPLC/Q-TOF-MS of Triterpenic Saponins From Chenopodium Quinoa Seeds. J Agric Food Chem. 60, 3113-3118, DOI: https://doi.org/10.1021/jf205010c (2012).

4. Yao, Y., Yang, X., Shi, Z. \& Ren, G. Anti-Inflammatory Activity of Saponins From Quinoa (Chenopodium Quinoa Willd.) Seeds in Lipopolysaccharide-Stimulated RAW 264.7 Macrophages Cells. J FOOD SCl. 79, H1018-H1023, DOI: https://doi.org/10.1111/1750-3841.12425 (2014).

5. Yao, Y. et al. Suppressive Effects of Saponin-Enriched Extracts From Quinoa On 3T3-L1 Adipocyte Differentiation. FOOD FUNCT. 6, 3282-3290, DOI: https://doi.org/10.1039/c5fo007 16j (2015).

6. Kuljanabhagavad, T., Thongphasuk, P., Chamulitrat, W. \& Wink, M. Triterpene Saponins From Chenopodium Quinoa Willd. PHYTOCHEMISTRY. 69, 1919-1926, DOI: https://doi.org/10.1016/ j.phytochem.2008.03.001 (2008).

7. Verza, S. G. et al. Immunoadjuvant Activity, Toxicity Assays, and Determination by UPLC/Q-TOF-MS of Triterpenic Saponins From Chenopodium Quinoa Seeds. J Agric Food Chem. 60, 3113-3118, DOI: https://doi.org/10.1021/jf205010c (2012).

8. Graf, B. L. et al. Quinoa Seeds Leach Phytoecdysteroids and Other Compounds with Anti-Diabetic Properties. FOOD CHEM. 163, 178-185, DOI: https://doi.org/10.1016/j.foodchem.2014.04.088 (2014).

9. Meyer, B. N., Heinstein, P. F., Burnof-Radosevich, M., Delfel, N. E. \& McLaughlin, J. L. BioactivityDirected Isolation and Characterization of Quinoside a: One of the Toxic/Bitter Principles of Quinoa 
Seeds (Chenopodium Quinoa Willd.). J AGR FOOD CHEM. 38, 205-208, DOI:

https://doi.org/10.1021/jf00091a045 (1990).

10. Yao, Y., Yang, X., Shi, Z. \& Ren, G. Anti-Inflammatory Activity of Saponins From Quinoa (Chenopodium Quinoa Willd.) Seeds in Lipopolysaccharide-Stimulated RAW 264.7 Macrophages Cells. J FOOD SCI. 79, H1018-H1023, DOI: https://doi.org/10.1111/1750-3841.12425 (2014).

11. Tang, Y. \& Tsao, R. Phytochemicals in Quinoa and Amaranth Grains and their Antioxidant, AntiInflammatory, and Potential Health Beneficial Effects: A Review. MOL NUTR FOOD RES. 61, DOI: https://doi.org/10.1002/mnfr.201600767 (2017).

12. Palombini, S. V. et al. Evaluation of Nutritional Compounds in New Amaranth and Quinoa Cultivars. Ciência E Tecnologia De Alimentos. 33, 339-344, DOI: http://dx.doi.org/10.1590/S010120612013005000051 (2013).

13. Estrada, A., Li, B. \& Laarveld, B. Adjuvant Action of Chenopodium Quinoa Saponins On the Induction of Antibody Responses to Intragastric and Intranasal Administered Antigens in Mice. Comp Immunol Microbiol Infect Dis. 21, 225-236, DOI: https://doi.org/10.1016/s0147-9571(97) 00030-1 (1998).

14. Gee, J. M. et al. Saponins of quinoa (Chenopodium quinoa): effects of processing on their abundance in quinoa products and their biological effects on intestinal mucosal tissue. Journal of the Science of Food \& Agriculture. 63, 201-209, DOI: https://doi.org/10.1002/jsfa.2740630206 (2010).

15. Leon-Roque, N. et al. A green analytical assay for the quantitation of the total saponins in quinoa (Chenopodium quinoa Willd.) based on macro lens-coupled smartphone. TALANTA. 204, 576-585, DOI: https://doi.org/10.1016/j.talanta.2019.06.014 (2019).

16. Thimmappa, R., Geisler, K., Louveau, T., O'Maille, P. \& Osbourn, A. Triterpene Biosynthesis in Plants. ANNU REV PLANT BIOL. 65, 225-257, DOI: https://doi.org/10.1146/annurev-arplant-050312-120229 (2014).

17. Moses, T., Pollier, J., Thevelein, J. M. \& Goossens, A. Bioengineering of Plant (Tri)terpenoids: From Metabolic Engineering of Plants to Synthetic Biology in Vivo and in Vitro. The New phytologist. 200, 27-43, DOI: https://doi.org/10.1111/nph.12325 (2013).

18. Haralampidis, K., Trojanowska, M. \& Osbourn, A. E. Biosynthesis of Triterpenoid Saponins in Plants. 31-49, DOI: https://doi.org/10.1007/3-540-44604-4_2 (2002).

19. Tansakul, P., Shibuya, M., Kushiro, T. \& Ebizuka, Y. Dammarenediol-II Synthase, the First Dedicated Enzyme for Ginsenoside Biosynthesis, in Panax Ginseng. FEBS LETT. 580, 5143-5149, DOI: https://doi.org/10.1016/j.febslet.2006.08.044 (2006).

20. Phillips, D. R., Rasbery, J. M., Bartel, B. \& Matsuda, S. P. Biosynthetic Diversity in Plant Triterpene Cyclization. CURR OPIN PLANT BIOL. 9, 305-314, DOI: https://doi.org/10.1016/ j.pbi.2006.03.004 (2006).

21. Fiallos-Jurado, J. et al. Saponin Determination, Expression Analysis and Functional Characterization of Saponin Biosynthetic Genes in Chenopodium Quinoa Leaves. Plant science: an international 
journal of experimental plant biology. 250, 188-197, DOI: https://doi.org/10.1016/ j.plantsci.2016.05.015 (2016).

22. Jarvis, D. E. et al. The Genome of Chenopodium Quinoa. NATURE. 542, 307, DOI: https://doi.org/10.1038/nature21370 (2017).

23. Zou, C. et al. A High-Quality Genome Assembly of Quinoa Provides Insights Into the Molecular Basis of Salt Bladder-Based Salinity Tolerance and the Exceptional Nutritional Value. CELL RES. 27, 13271340, DOI: https://doi.org/10.1038/cr.2017.124 (2017).

24. Yu, G., Wang, L. G., Han, Y. \& He, Q. Y. ClusterProfiler: An R Package for Comparing Biological Themes Among Gene Clusters. OMICS. 16, 284-287, DOI: https://doi.org/10.1089/omi.2011.0118 (2012).

25. Vogler, B. K., Pittler, M. H. \& Ernst, E. The Efficacy of Ginseng. A Systematic Review of Randomised Clinical Trials. EUR J CLIN PHARMACOL. 55, 567-575, DOI: https://doi.org/ 10.1007/s002280050674 (1999).

26. Shibata, S. Chemistry and Cancer Preventing Activities of Ginseng Saponins and some Related Triterpenoid Compounds. J KOREAN MED SCl. 16 Suppl, S28-S37, DOI: https://doi.org/ 10.3346/jkms.2001.16.S.S28 (2001).

27. Matzke, M. A. \& Mosher, R. A. RNA-directed DNA Methylation: An Epigenetic Pathway of Increasing Complexity. NAT REV GENET. 15, 394-408, DOI: https://doi.org/10.1038/nrg3683 (2014).

28. Kim, D. \& Sung, S. Vernalization-Triggered Intragenic Chromatin Loop Formation by Long Noncoding RNAs. DEV CELL. 40, 302-312, DOl: https://doi.org/10.1016/j.devcel.2016.12.021 (2017).

29. Li, S., Yamada, M., Han, X., Ohler, U. \& Benfey, P. N. High-Resolution Expression Map of the Arabidopsis Root Reveals Alternative Splicing and lincRNA Regulation. DEV CELL. 39, 508-522, DOI: https://doi.org/10.1016/j.devcel.2016.10.012 (2016).

30. Wang, Y. et al. Arabidopsis Noncoding RNA Mediates Control of Photomorphogenesis by Red Light. Proceedings of the National Academy of Sciences. 111, 10359-10364, DOI: https://doi.org/ 10.1073/pnas.1409457111 (2014).

31. Di C et al. Characterization of Stress-Responsive IncRNAs in Arabidopsis Thaliana by Integrating Expression, Epigenetic and Structural Features. PLANT J. 80, 848-861, DOI: https://doi.org/ 10.1111/tpj.12679 (2014).

32. Wang, D. et al. Transposable Elements (TEs) Contribute to Stress-Related Long Intergenic Noncoding RNAs in Plants. The Plant Journal. 90, 133-146, DOI: https://doi.org/ 10.1111/tpj.13481 (2017).

33. Zhang, Y. C. et al. Genome-wide screening and functional analysis identify a large number of long noncoding RNAs involved in the sexual reproduction of rice. GENOME BIOL. 15, 512, DOI: https://doi.org/10.1186/s13059-014-0512-1 (2014).

\section{Figures}




\section{RNA-seq raw data}

(four groups of quinoa samples)

\section{QC\&Quantification}

\section{Differential expression analysis}

\section{gene set enrichment analysis(GSEA)}

\section{Principal component analysis (PCA)}

\section{Key genes}

Figure 1

Flowchart of screening for the key genes associated with the metabolism of quinoa saponins. QC: quality control; DEGs: differential expression genes. 

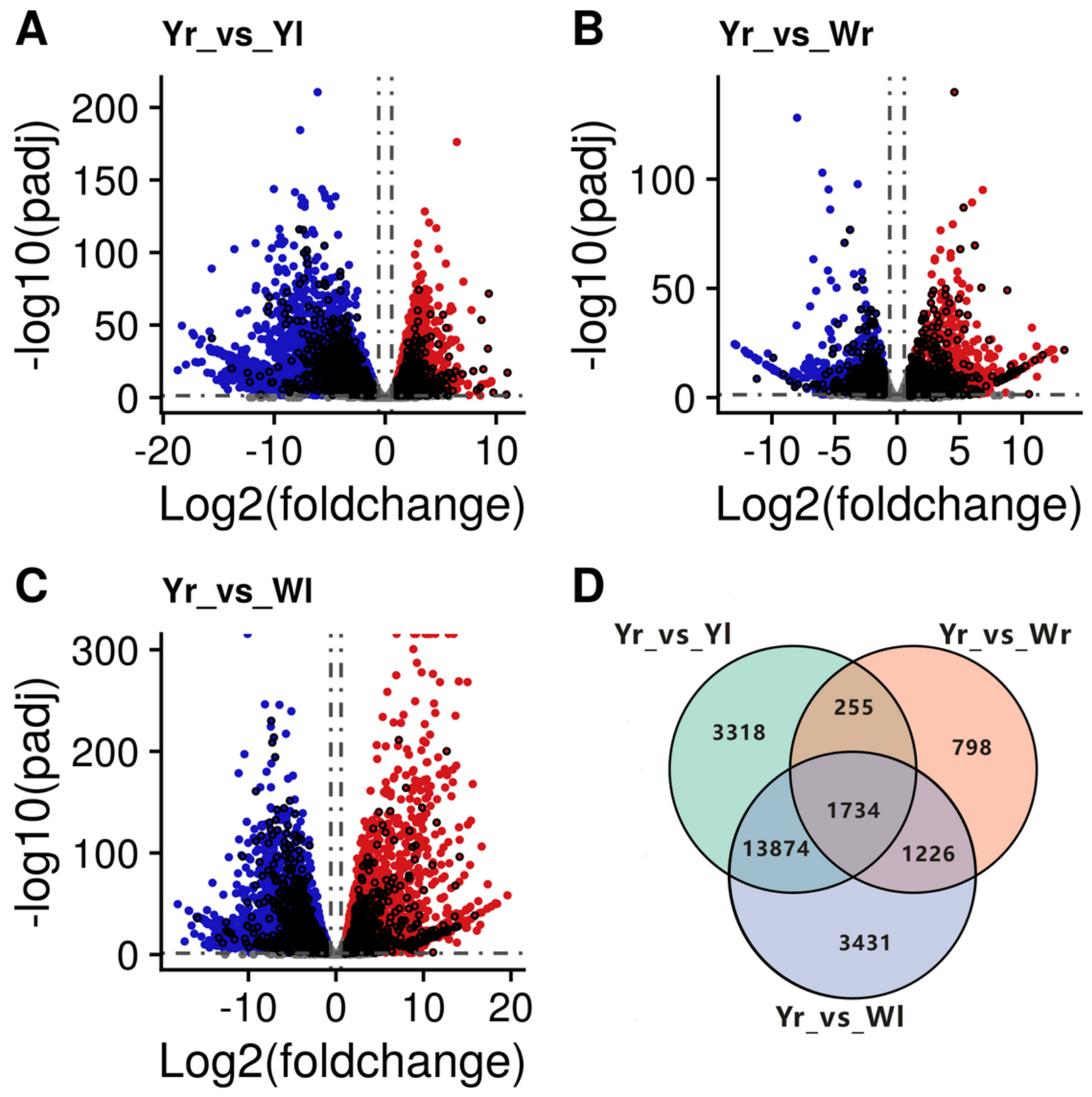

D

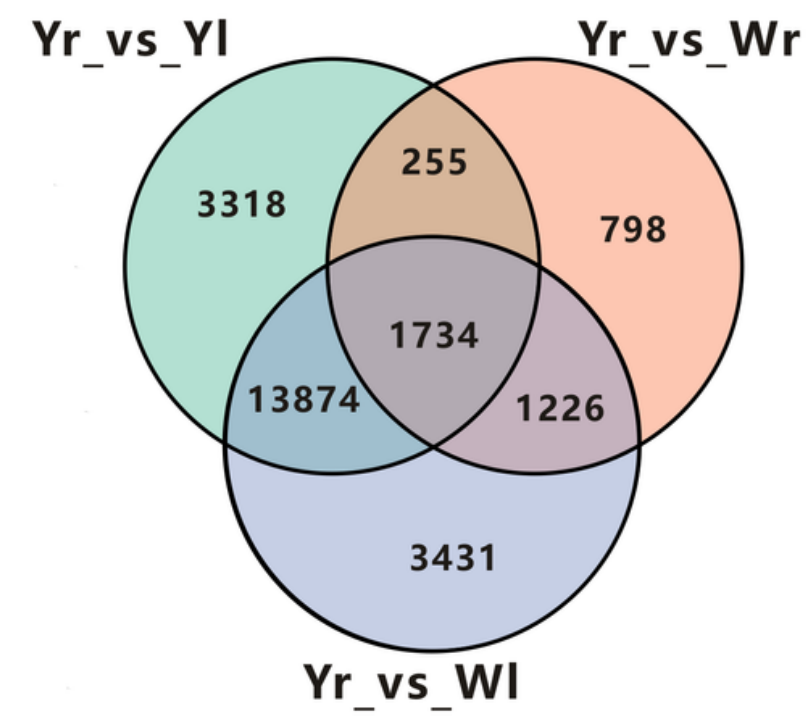

Figure 2

Expression differences between three pair of sample groups. (a) Volcano plot of the DEGs between $\mathrm{Yr}$ and Wr. (b) Volcano plot of the DEGs between Yr and YI. (c) Volcano plot of the DEGs between Yrand WI. (d) Venn diagrams of DEGs for three DEGs sets. Red dots: up-regulated genes. Blue dots: down-regulated genes. Black circle in A, B, C: the genes obtained by taking the intersection of three DEGs set. "padj": corrected $p$ values. 

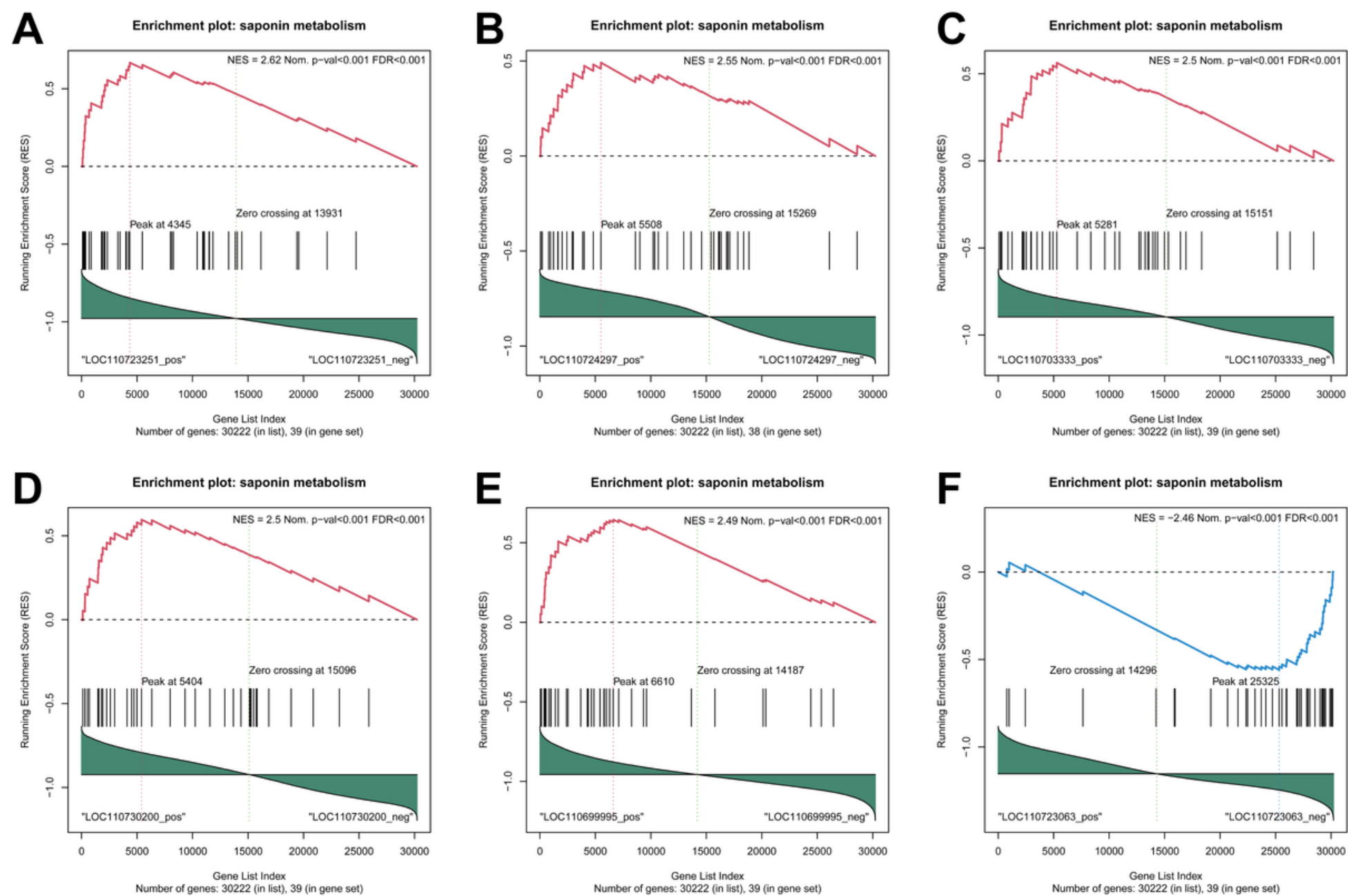

Figure 3

Genes related to saponin metabolism pathways. (a-f) The first six genes (LOC110723251, LOC110724297, LOC110703333, LOC110730200, LOC110699995, LOC110723063) most closely associated with saponin metabolism. "pos": Positive correlation; "neg": negative correlation; "NES": Normalized Enrichment Score; "Nom P-val”: normalized P value; "FDR": false discovery rate. 

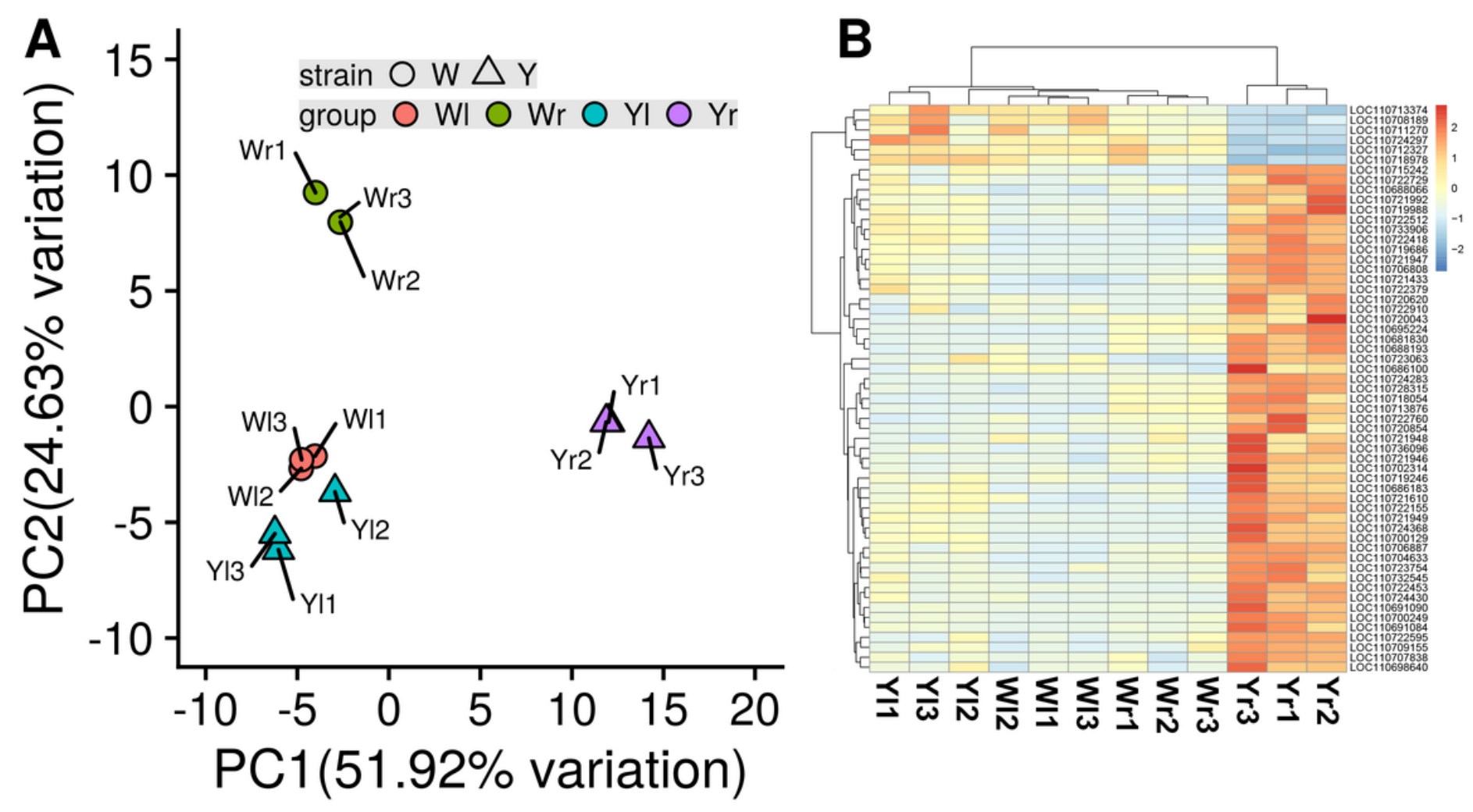

\section{Figure 4}

Dimensionality reduction of key candidate genes. (a) Principal components analysis (PCA) of key candidate genes; (b) clustering heatmap of 57 key genes in the quinoa. Yr: yellow quinoa fruit; Yl: yellow quinoa flower; Wr: white quinoa fruit; Wl: white quinoa flower. The number that follows sample names represent three biological repeats.

Gene_type $\square$ IncRNA $\square$ protein_coding

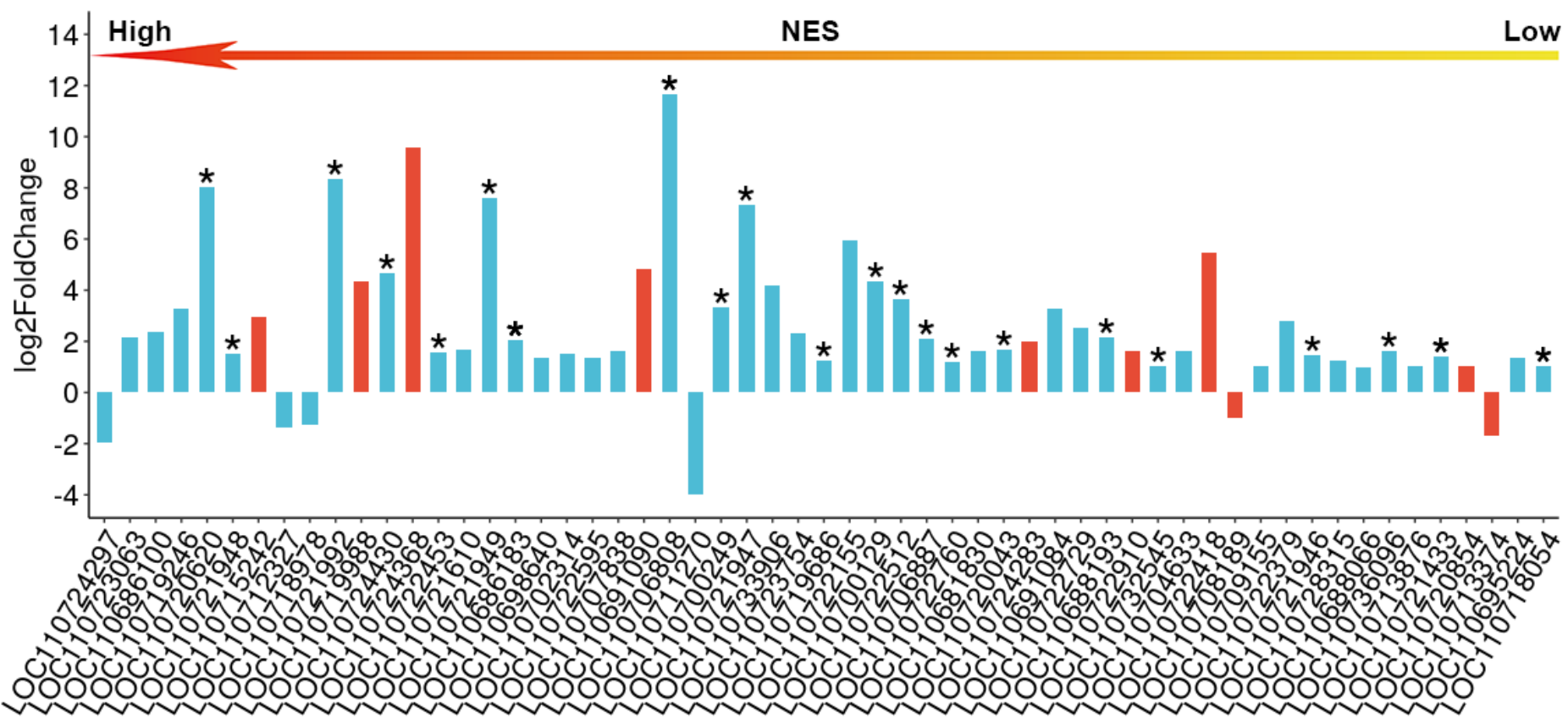


Figure 5

The details on expression pattern of 57 key genes related to saponin metabolism. NES: normalized enrichment score; ' ${ }^{\prime}$ ': uncharacterized protein.

\section{Supplementary Files}

This is a list of supplementary files associated with this preprint. Click to download.

- TableS1.xlsx

- TableS2.xIsx

- TableS3.xIsx

- supplementary.docx 